\title{
Crossing barriers from blood-to-brain and academia-to-industry
}

\author{
"Adhering to the ten key development criteria presented in this paper will hopefully allow for faster and more successful \\ development of treatments for devastating brain diseases."
}

Keywords: blood-brain barrier $\approx$ brain disease $\approx$ drug delivery $\approx$ drug targeting $₫$ neuroscience

\section{Crossing the blood-brain barrier}

The worldwide economic burden caused by CNS diseases has reached over US $\$ 2$ trillion a year according to the Neuroinsights' Neurotechnology Industry 2008 Report [1]. This burden will continue to rise when taking the current demographic development of an increasingly aging population into account. However, treatments for the majority of CNS diseases are not yet widely available, in large part due to the presence of the neuroprotective blood-brain barrier (BBB), and (therefore) a lack of validated targets for these CNS diseases. Most drugs currently in development against neurological targets show high affinity for the target. However, they often have either poor BBB penetration or a very low solubility (or both), resulting in cessation of their development for reasons such as lack of therapeutic efficacy, dose-limiting toxicity outside the CNS or off-target effects.

Although there are many routes for drug delivery to the brain, only drug delivery via the vascular route will allow for a widespread diffusion of the infused drug throughout the whole brain due to the large surface area of the human BBB [2]. In fact, almost every neuron has its own brain capillary for oxygen and nutrient supply [3]. This makes the vascular route a very promising one for drug delivery to the brain, especially since delivery throughout wide parts of the human brain more often than not seems essential to effectively modify CNS disorders. This even appears to hold true for disorders that are often considered a local disease such as Parkinson's disease, Huntington's disease or brain cancer.

Basically, two methods have been described in the literature to actively enhance drug delivery to the brain after systemic administration: either opening/disruption of the neuroprotective BBB by osmotic imbalance, ultrasound or vasoactive compounds (e.g., bradykinin or P-glycoprotein inhibitors), or physiological strategies aiming to use endogenous transport mechanisms. While the first method has the disadvantage that neurons may be damaged (semi)-permanently due to unwanted blood components entering the brain [4], the physiological strategies have a large potential as discussed in several review papers elsewhere [5-11]. As a third alternative (using a combination of aspects of both methods), positive charge has also been applied to compounds or drug carriers to quite effectively enhance the absorptive-mediated transport across the BBB $[12,13]$; however, a beneficial therapeutic window of this basically toxic transport mechanism has thus far not been established.

Based on the above, it is our vision that safely enhancing the blood-to-brain delivery of drugs is ultimately best achieved by targeted physiologically based brain drug delivery systems that fully respect and maintain BBB integrity.

\section{Crossing the}

\section{academia-industry barrier}

Many brain drug delivery systems targeting the transferrin receptor, insulin receptor, low density lipoprotein receptor-related protein (LRP) receptors, diphtheria toxin receptor and more recently also glutathione transporters, have been widely investigated in academic settings for well over a decade, if not longer. In some cases this research from academic research groups was successful enough that patents were filed and sometimes even licensed to pharmaceutical companies, or spin-off companies were created. However, overall success is still limited as can be judged from the few drugs that are

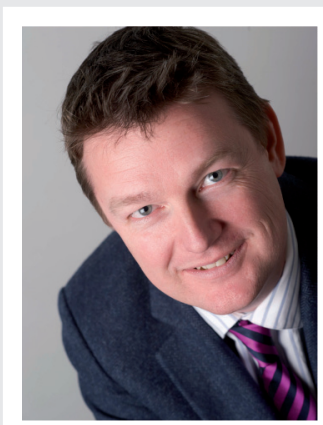

Pieter J Gaillard

to-BBB technologies BV, Bio Partner Center II, Niels

Bohrweg II, Bio-Science Park Leiden, 2333 CA Leiden,

The Netherlands

Tel.: +31 713322 255

Fax: +31848313409

E-mail: gaillard@toBBB.com 
being developed for clinical use, and none of these systems have yet been approved for use in patients, despite the large unmet medical needs for CNS diseases.

For a long time, pharmaceutical industries have shied away from the development of efficient (intravenously applied) brain delivery systems, instead the industry has relied exclusively on medicinal chemistry and screening of large libraries of (orally available) small molecules to provide the drug candidates they preferred. However, despite all efforts, $98 \%$ of the small molecules are not able to effectively cross the BBB within an acceptable therapeutic window [14]. Furthermore, most CNS diseases are complex disorders with difficult molecular targets, also warranting the discovery and development of the inherently more safe and selective biological drugs, such as enzymes, proteins, or nucleic acid-based molecules. The BBB is, however, even more impermeable to such large molecules and local delivery proved to be a difficult approach to translate from laboratory to clinic. As a result, again, this approach has not been able to serve the many unmet medical needs that are still present in CNS disorders and new developments are desperately needed.

So, crossing the BBB as well as the academiaindustry barrier has thus far proven to be a formidable challenge, especially because the technological requirements to do so have historically been very much detached from the needs of pharmaceutical companies, where academic researchers are often only focused and trained on limited aspects of drug delivery to the brain and not on the full drug development path. Nowadays, the industry's acceptance for more complex brain drug delivery systems is growing in order to address the large growth market of un(der)treated CNS disorders, which is mainly driven by the medical and commercial success of parenterally administered biologics.

In order to further improve the transfer from academically discovered CNS drug targeting approaches to commercially viable products, we propose to adhere to ten key development criteria for the creation of industry-proof targeted blood-to-brain drug delivery systems. These criteria are related to safely targeting the $\mathrm{BBB}$, the use of drug carriers, and the path to progress these systems from laboratory to clinic (TABLE I). Adhering to the ten key development criteria presented in this paper will hopefully allow for faster and more successful development of treatments for devastating brain diseases. The G-Technology ${ }^{\circledR}$, recently developed by to-BBB, will be used to illustrate these key criteria. In fact, the G-Technology is currently being evaluated by a growing list of top tier pharmaceutical companies to establish a potential technological and therapeutical match with their molecules of interest in their disease models [201].

\section{Glutathione-coated liposomes for enhanced blood-to-brain drug delivery (G-technology)}

Glutathione is an endogenous tripeptide that possesses antioxidant-like properties and plays a central role in the detoxification of intracellular metabolites; it has specific and active uptake transporters expressed at the BBB [15-17]. Based on these properties and on previous unpublished validation results from Maggie Lu (at the Industrial Technology Research Institute [ITRI] in Hsin-Chu, Taiwan, Republic of China), ITRI was the first to file patents describing glutathionemediated drug delivery to the brain [101]. In 2008, to-BBB technologies BV obtained the exclusive worldwide rights to commercialize these patents for the targeted delivery of drugs to the brain.

In several proof-of-concept studies we and ITRI have demonstrated that glutathione-PEG liposomes loaded with peptides and small molecules in several pain models, brain tumors and viral encephalitis, safely enhanced the delivery of these drugs to the brain, thereby decreasing pain sensitivity, inhibiting tumor growth and prolonging survival [18], and reducing the lethal effects of viral infections in the brain, respectively.

Recently, we have also shown that liposomes coated with glutathione-conjugated PEG are able to deliver free drug to the brain, as measured by microdialysis [19]. In this mechanistic study, liposomes coated with increasing numbers of glutathione molecules conjugated to PEG on the outside of the liposomes were injected intravenously; the liposomes encapsulated ribavirin as an example drug. The average plasma halflife of the liposomes was approximately $19 \mathrm{~h}$; at the tested conditions glutathione coating did not have a significant effect on the plasma clearance of the liposomal ribavirin, although the highest level of glutathione coating displayed a trend towards faster plasma clearance. In contrast, increasing amounts of glutathione coating resulted in higher amounts of free ribavirin in the brain, providing mechanistic data that glutathione enhanced the delivery of free drug across the BBB [19]. 
Ten key development criteria for targeted blood-to-brain drug delivery

- Proven inherently safe receptor biology in humans

For a long time, research has focused on receptors for drug delivery across the BBB. Not all receptors are inherently suitable for drug targeting to the brain as they may have an important biological function that should not be interfered with too much. Saturation with exogenous ligand may lead to obstruction of brain transport of essential endogenous compounds, resulting in a deprivation of the brain of these compounds. In addition, many receptors at the BBB have signaling functions upon binding of various ligands. Interference with such signaling functions by drug delivery systems is difficult to ascertain in rodents, let alone humans, especially if disease progression is also to be taken into account. Therefore, starting the development of systems that target inherently safe receptors will greatly facilitate the translation into clinical or commercial products.

The glutathione transporter has a good safety profile in humans, since the physiological plasma and brain concentrations of glutathione are high, in the millimolar range. Furthermore, the $\mathrm{K}_{\mathrm{m}}$ for the transporter at the BBB was reported to be high, also in the millimolar range [16]. The low (micromolar) concentrations used for drug targeting are, therefore, not likely to be interfering with the physiological function of the glutathione transporter. The potential signaling function of glutathione-sensitive receptors at the level of the BBB, however, remains to be elucidated.

\section{Safe \& human-applicable ligand}

Endogenous ligands as targeting vectors are undoubtedly safe; however, these often cannot be used due to competition with high levels in plasma. The safety of other targeting vectors will need some attention as these might interfere with the endogenous ligand, and ultimately receptor function. The ultimate goal of any drug development program at the pharmaceutical industry is to deliver safe and effective human therapies, so obviously the ligands being developed in academia have to adhere to both criteria to be of any interest for commercial development.

Glutathione as a targeting vector has an excellent safety profile; it is already used as supportive therapy in chemotherapy [20] and as a food supplement. In addition, due to its small size, it is not expected to cause an immunologic response.
Table 1. Ten key development criteria for targeted blood-to-brain drug delivery.

\begin{tabular}{|lll|}
$\begin{array}{l}\text { Targeting the } \\
\text { blood-brain barrier }\end{array}$ & Drug carriers & $\begin{array}{l}\text { Drug development from } \\
\text { laboratory to clinic }\end{array}$ \\
$\begin{array}{l}\text { Proven inherently safe } \\
\text { receptor biology in } \\
\text { humans }\end{array}$ & $\begin{array}{l}\text { No modification of } \\
\text { active ingredient }\end{array}$ & $\begin{array}{l}\text { Low costs and } \\
\text { straightforward } \\
\text { manufacturing }\end{array}$ \\
$\begin{array}{l}\text { Safe and human } \\
\text { applicable ligand }\end{array}$ & $\begin{array}{l}\text { Able to carry various } \\
\text { classes of molecules }\end{array}$ & Activity in all animal models \\
$\begin{array}{lll}\text { Receptor-specific binding } & & \text { Strong intellectual } \\
\text { property protection }\end{array}$ \\
$\begin{array}{l}\text { Applicable for acute and } \\
\text { chronic indications }\end{array}$ & \\
\hline \multicolumn{2}{|c|}{ Favorable pharmacokinetics } & \\
\hline
\end{tabular}

- Receptor-specific binding

The binding of the targeting ligand to the receptor needs to be specific to limit unwanted side effects. A deep understanding of the specificity of a newly discovered targeted system will lead to better predictability during further development of the system and products based thereon, and therefore get a better response from the pharmaceutical industry. Most targeting vectors are specific for their target receptors as this is one of the first properties to be investigated. In addition, the organ specificity of the ligand-receptor interaction is also preferentially or exclusively in the CNS, or more specifically, at the BBB. For most of the academically developed systems this is, however, not the case; the insulin and transferrin receptors, as well as the LRP receptors, are widely expressed throughout the entire body, including the brain.

Although many cells in the body are able to take up glutathione through facilitated transport, only in the CNS and BBB have glutathione transporters been described that can actively (sodium-dependently) accumulate and transport intact glutathione against a concentration gradient [15-17].

\section{- Applicable for acute \& chronic indications} When applying targeting strategies, the diseased state should be considered as the receptor expression levels or function might be changed [11]. Not only is the efficacy of the selected target receptor and ligand important in view of the disease, safety is also important. For chronic conditions such as Alzheimer's or Parkinson's disease, a continuous treatment is necessary and, thus, the safety should be warranted for a prolonged time, while for acute indications such as encephalitis a different safety profile is warranted. For obvious 
commercial reasons, the ultimate interest from the pharmaceutical industry is by developing treatments for chronic CNS disorders. Academic drug-delivery systems that lack the outlook to be chronically applied will therefore have difficulties in being taken up by the industry and obtaining the required funding.

Glutathione is the only endogenous targeting vector with a known safety profile for prolonged exogenous administration, since - as previously mentioned - it is already in use as a food supplement and as supportive therapy in chemotherapy [20]. To date, not enough data have been published to judge the long-term safety of administration of other exogenous ligands used as targeting vectors.

\section{- Favorable pharmacokinetics}

Next to an efficient and safe targeting strategy, a favorable pharmacokinetic (or toxicokinetic) profile of these intravenously applied drug delivery systems is also important, as a prolonged circulation in plasma will allow sufficient time to reach and maintain presence at the target site. Patients simply cannot be on a continuous infusion for several days or week in a row, if the mechanism of action of the drug should require that. Drug carriers, such as liposomes that are used in the G-Technology, often have this capacity due to the protective PEG coating [21,22]. In humans, indeed a half-life of several days has been reported for PEGylated liposomes with the anticancer drug doxorubicin $\left(\right.$ Doxil $^{\circledR}$, Caelyx $\left.^{\circledR}\right)$, allowing for a 3-to-4-week cycle of treatments (also taking the required recovery period into account from the chemotherapeutic drug).

In addition to improving the pharmacokinetic properties of an encapsulated drug in a drug carrier by protecting the drug from metabolism and elimination in the systemic circulation, encapsulated compounds are also better shielded from the immune system and drug carriers, thereby helping to prevent immunogenic reactions to the compounds. In line with this, the off-target sites of the compound are also often reduced by encapsulation in drug carriers. The classic example for this is the significant reduction of cardiotoxicity for liposomal doxorubicin (Doxil, Caelyx), which is otherwise the dose-limiting toxicity for free doxorubicin.

\section{- No modification of active ingredient}

In contrast to direct conjugates of drug and targeting vector (or fusion proteins thereof), drug molecules do not need to be modified to be incorporated into liposomes or other drug carriers. Drug modification, either via conjugation or through expression of fusion proteins, might result in a loss of activity [23], which can be avoided by using liposomes or nanoparticles. In addition, an immunogenic response might occur against the newly formed fusion proteins or protein conjugates. Finally, the drug development process is more predictable when drugs are not modified; once the unmodified drugs from liposomes or nanoparticles are released at the target site and present in cells or tissues, one can continue to use and compare to the already available data on the drug of interest. As an example, available data on the mechanism of action, pharmacokinetics and pharmacodynamics of doxorubicin and liposomal doxorubicin (Doxil, Caelyx) are helpful for the development of brain-targeted doxorubicin liposomes (which is to-BBB's lead product based on the G-Technology, currently in development for brain cancer).

On another note, pharmaceutical companies are very reluctant to modify their proprietary compounds in collaboration with third parties, including academia, making investments in such projects legally very difficult to establish in a mutually satisfactory structure.

- Able to carry various classes of molecules While direct linking strategies are suitable for some proteins, peptides and certain synthetic molecules - all depending on the suitability of the specific linker strategies, chemistries and biological compatibility - in most cases they cannot be efficiently used for small molecules and many nucleic acid-based drugs. Liposomes or nanoparticles can potentially carry all these classes without the need for modification of the active molecules, making these drug-carrier systems a faster and more likely to succeed strategy for new projects.

A more versatile drug-delivery system also justifies a broader investment in the platform, as the pay-off will be beneficial to multiple potential products.

Low costs \& straightforward manufacturing To bring a drug to the market, straightforward manufacturing and low (or justifiable) costs are favorable, if not essential. Straightforward manufacturing will ensure a smooth and predictable development process. For conjugates prepared by chemical linkage it is important to demonstrate a consistent product 
and manufacturing process, especially from a regulatory viewpoint. Although for a single product, fusion proteins expressed in cell systems become more and more affordable, every new product will need to be completely newly designed and generated, with all development risks and costs associated. So, all newly developed systems will need to overcome significant chemistry, manufacturing and controls (CMC) regulatory hurdles, which require significant investment in the pharmaceutical development processes. Often such investments are not available to academic institutions through the regular sources of funding and the pharmaceutical industry is rightfully reluctant to take such development risks upfront without significant proof-of-concept with their molecule of interest, preferably in their own hands. Building a new drug-delivery system on established technologies will therefore enable a smoother transfer to the pharmaceutical industry.

The tripeptide glutathione used in the G-Technology has limited possibilities for linking or conjugation to the drug or drug carrier and therefore leads to a more straightforward CMC development process. In addition, starting the clinical development of the first lead product based on a marketed PEGylated liposomal product (Doxil, Caelyx) has significantly improved the chances of success for the CMC development process, and thereby also lowered the costs of manufacturing to a justifiable level.

\section{Activity in all animal models}

Although it may read as trivial, in practice it is not: drug development is further facilitated if the technology has activity in all mammalian models, including humans. It is then possible to select already established models for early proof-of-concept and safety studies allowing for a faster development. In contrast to the humanspecific targeting antibodies raised against the human insulin receptor, the selected ligands for the LRP receptors, as well as for the glutathione transporters, are conserved across all species. Thus it is possible to select any animal model for investigational studies and use the same product for the CMC and clinical development phase.

\section{- Strong intellectual property protection}

Finally, strong intellectual property protection is needed to commercially exploit the drug. The obviously large investments required developing any targeted drug delivery system into a successful new treatment option for CNS disorders need to be well protected from the start. There are no commercial incentives to invest in such extensive developments if the parties investing their resources into these developments do not own the basic technologies. Therefore, patent protection before publishing is of paramount importance to further technologies in this nascent field.

\section{Future perspective}

Clearly, the momentum is gaining for the development of new technologies to enhance the delivery of drugs to the brain within a beneficial therapeutic window. On the one hand, academic research in this field has provided more and more promising targeting approaches and drug-delivery technologies, whereas on the other hand, the pharmaceutical industry has welcomed the more complex brain drug delivery systems to address the large growth market of un(der)treated CNS disorders. Having said that, a clear understanding of the ground to be covered between academic research and commercially viable products will be essential to make optimal use of this momentum. With the ten key development criteria discussed in this paper, we hope to add to bridging this gap so that more and more patients suffering from devastating CNS disorders will be better served in the distant future.

\section{Executive summary}

- Treatments for the majority of CNS diseases are not yet widely available, in large part due to the presence of the neuroprotective blood-brain barrier and (therefore) a lack of validated targets for these CNS diseases.

- It is our vision that treatment of brain diseases will be best achieved by safely enhancing the bloodto-brain delivery of drugs.

- In order to further improve the transfer from academically discovered CNS-targeted drug delivery systems to commercially viable approaches, ten key development criteria are discussed relating to safely targeting the blood-brain barrier, the use of drug carriers, and the path to progress of these systems from laboratory to clinic. 


\section{Acknowledgements}

Pieter Gaillard acknowledges Aad van Vliet/ TwoMoves for the author photograph (@) Aad van Vliet/TwoMoves).

\section{Financial \& competing interests disclosure}

Pieter Gaillard is to-BBB's Chief Scientific Officer, and holds founder shares in to-BBB technologies $B V$. The author has no other relevant affiliations or financial involvement with any organization or entity with a financial interest in or financial conflict with the subject matter or materials discussed in the manuscript apart from those disclosed.

Corine Visser (publication program manager at to-BBB technologies $B V$ ) has provided writing and editorial assistance.

\section{Bibliography}

1 The Neurotechnology Industry 2008 Report. Pub ID: NEI1781046. NeuroInsights, LLC. May 8 (2008).

2 Pardridge WM. Drug and gene delivery to the brain: the vascular route. Neuron 36(4), 555-558 (2002).

3 Gaillard PJ, Visser CC, de Boer AG. Targeted delivery across the blood-brain barrier. Expert Opin. Drug Deliv. 2(2), 299-309 (2005).

4 Bellavance MA, Blanchette M, Fortin D. Recent advances in blood-brain barrier disruption as a CNS delivery strategy. AAPS. J. 10(1), 166-177 (2008).

5 de Boer AG, Gaillard PJ. Drug targeting to the brain. Annu. Rev. Pharmacol. Toxicol. 47, 323-355 (2007).

6 de Boer AG, van der Sandt IC, Gaillard PJ. The role of drug transporters at the blood-brain barrier. Annu. Rev. Pharmacol. Toxicol. 43, 629-656 (2003).

7 Gabathuler R. Approaches to transport therapeutic drugs across the blood-brain barrier to treat brain diseases. Neurobiol. Dis. 37(1), 48-57 (2010).
8 Jones AR, Shusta EV. Blood-brain barrier transport of therapeutics via receptor-mediation. Pharm. Res. 24(9), 1759-1771 (2007).

9 Pardridge WM. Brain drug development and brain drug targeting. Pharm. Res. 24(9), 1729-1732 (2007).

10 Pardridge WM. Drug targeting to the brain. Pharm. Res. 24(9), 1733-1744 (2007).

11 Rip J, Schenk GJ, de Boer AG. Differential receptor-mediated drug targeting to the diseased brain. Expert Opin. Drug Deliv. 6(3), 227-237 (2009).

12 Bickel U, Yoshikawa T, Pardridge WM. Delivery of peptides and proteins through the blood-brain barrier. Adv. Drug Deliv. Rev. 46(1-3), 247-279 (2001).

$13 \mathrm{Lu} \mathrm{W,} \mathrm{Wan} \mathrm{J,} \mathrm{She} \mathrm{Z,} \mathrm{Jiang} \mathrm{X.} \mathrm{Brain} \mathrm{delivery}$ property and accelerated blood clearance of cationic albumin conjugated pegylated nanoparticle. J. Control. Release 118(1), 38-53 (2007).

14 Pardridge WM. Blood-brain barrier drug targeting: the future of brain drug development. Mol. Interv. 3(2), 90-105 (2003).

15 Kannan R, Chakrabarti R, Tang D, Kim KJ, Kaplowitz N. GSH transport in human cerebrovascular endothelial cells and human astrocytes: evidence for luminal localization of $\mathrm{Na}^{+}$-dependent GSH transport in HCEC. Brain Res. 852(2), 374-382 (2000).

16 Kannan R, Kuhlenkamp JF, Jeandidier E et al. Evidence for carriermediated transport of glutathione across the blood-brain barrier in the rat. J. Clin. Invest. 85(6), 2009-2013 (1990).

17 Zlokovic BV, Mackic JB, McComb JG et al. Evidence for transcapillary transport of reduced glutathione in vascular perfused guinea-pig brain. Biochem. Biophys. Res. Commun. 201(1), 402-408 (1994).

18 van Tellingen $\mathrm{O}$, Brandsma D, Appeldoorn CCM et al. GSH-conjugation improves efficacy of Doxil against intracranial xenografts. Presented at: 101st Annual Meeting of the American Association for Cancer Research. Washington DC, PA, USA, 17-21 April 2010.
19 Rip J, Appeldoorn CCM, Manca FM et al. Receptor-mediated delivery of drugs across the blood-brain barrier. Presented at: Pharmacology and Toxicology of the Blood-brain Barrier: State of the Art, Needs for Future Research and Expected Benefits for the EU. Brussels, Belgium, 11-12 February 2010.

20 Block KI, Koch AC, Mead MN et al. Impact of antioxidant supplementation on chemotherapeutic toxicity: a systematic review of the evidence from randomized controlled trials. Int. J. Cancer. 123(6), 1227-1239 (2008).

21 Brasnjevic I, Steinbusch HW, Schmitz C, Martinez-Martinez P. Delivery of peptide and protein drugs over the blood-brain barrier. Prog. Neurobiol. 87(4), 212-251 (2009).

22 Woodle MC, Lasic DD. Sterically stabilized liposomes. Biochim. Biophys. Acta 1113(2), 171-199 (1992).

23 Gaillard PJ, Visser CC, Appeldoorn CCM, Rip J. Blood-to-brain drug delivery - $10 \mathrm{key}$ development criteria. Curr. Pharm. Biotechnol. (2010) (In Press).

\section{Patent}

101 Industrial Technology Research Institute. US20070141133 (2005).

\section{- Website}

201 to-BBB technologies B.V: Dutch biotechnology company in the field of enhanced drug delivery across the blood-brain barrier www.toBBB.com 